the anastomosis shonld be closed. One is naturally reluc tant to recommend such a step, but so far as I have seen the results have always justified it. The constant nauser, which was a prominent symptom in all of them, was got rid of, and, although none of the patients was restored to perfect health, they were all greatly relieved and brought back to a condition of comparative efficiency.

The moral to be drawn from these disappointments is that cases for gastro-enterostomy should be selected with greater care than is always exercised, and the opinion of a physician as to the adrisability of the operation should be obtained as well as that of a surgeon. Even with the greatest care mistakes in diagnosis will occur, but the surgeon should have the moral courage to close the abdomen and proceed no. further if at the time of the operation no definite organic lesion of the stomach or duodenum can be demonstrated. In some of the cases I have seen there was strong reason to suspect that the operation had been -performed for atonic dilatation or gastroptosis only; the results are always bad. Even when an organic lesion is present the coexistence of decided atony of the stomach is a contraindication to operation unless careful and prolonged medical treatment has failed to relieve.

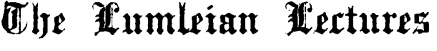

\section{CEREBRO-SPINAL FEVER.}

Delivened before the Royal College of Physicians of London,

BY

SIR HUMPHRY ROLLESTON, K.C.B., M.D., F.R.C.P. EMERITUS PHYSICIAN, ST. GEORGE'S HOSPITAI; PRESIDENT,

\section{LECTURE II.}

(Abstract.)

Is his second lecture Sir Humphry Rolleston gave clinical picture of the disease. The incubation period has been variously stated to be from one day up to as long an interval as thirty days, most writers inclining to a period of four or five days, and very few regarding.it as long as ten days. These estimates have been based on the interval between known contact with a case or a recognized carrier and the appearance of symptoms, but a more accurate basis for deciding the incubation period would be provided by determining the average duration of the period that meningococci are present in the nasopharynx before symptoms of systemic infection appear. Fildes and Baker examined with a negative result 26 men who subsequently contracted the disease, concluding that carriers seldom develop the disease, and that the period of time between the acquisition of the meningococcus and the onset of the disease must usually be short. Out of these 26 cases, 5 were examined within a week of the onset, 8 during the second week, and 4 in the third week before the onset.

The general opinion, as voiced by von Lingelsheim, Gordon, and Andrewes, is that meningococci are constantly present in the throat at the onset of the disease and that every case arises in a carrier. Fildes and Baker's observations, as far as they go, are certainly compatible with this view that the incubation period is very short, and need not be more than a day or two, but their failure to find any meningococci in the throats of persons who afterwards contracted the disease is excep tional, for other observers with much smaller series of cases have usually been able to point to some examples of known carriers contracting the disease; thus, among Martin Flack's 185 carriers 4 developed the disease during isolation.

ONSET.

The usual form is much like that of ordinary "influenza," and is sudden, with headache, fever, and vomiting, the symptoms becoming progressively worse, so that in twenty four hrours meningitis is suggested. Sometimes after this form of onset the symptoms rather suddeuly advance, so that in a short time-an hour or two-the patient is comatose or delirious; in young children, and occasionally in adults, the onset may be marked by convulsions. On the other laand, after the onset there may be a fallaciaus improvement for a day or two, as if the blood invasion were yielding to the forces of natural immunity, and then meningitic symptoms appear. The catarrhal onset lasting some days is more gradual.

The acute or fulminating onset is the least frequent; it may talse various forms. It may be maniacal, imitatin. delirium tremens, attended by convulsions suggesting epilepsy, by hallucinations or syncape, or the patient may be unconscious when first seen. In patients found unconscious, or in the apoplectic mode of onset, the diagnosis is obviously very difficult; in some instances the patient has fallen out of bed or out of his hammock, and the question of fractured base has arisen. 'I'his apoplectiform onset may be rapidly followed by death. and thus accounts for cases of sudden death unexplaineil until examination after death shows the presence of the meningococci in the central nervous system.

With severe haemic infection the manifestations are those of grave toxaemia, approaching the typhoid state, often at the outset with a subnormal temperature, which subsequently rises rapidly, quick pulse and respirations, vomiting, and a baemorrhagic rash. Sudden cardiac collapse and fatal syncope at the onset of meningitis are specially described by Sainton. The initial shock may be considerable, and when accompanied by vomiting, abdominal pain, and diarrhoea, may suggest an acute abdo. minal lesion such as appendicitis or perforation, and laparotomy has naturally been performed. One such case in the navy was operated upon for a supposed perforated gastric ulcer and found to have a normal abdomen; at the suggestion of the anaesthetist, lumbar puncture was at once performed, and turbid fluid containing meningococci obtained.

\section{Clinical Forms.}

Cerebro'spinal fever, like acute poliomyelitis, has two distinct phases: (a) The general or systemic infection; and $(b)$ the localization in the meninges. The evidence of systemic infection is usually prominent in the initial stages, and the process is then commonly extremely acute, but it may continue for weeks, and in rather rare instances chronic septicaemia follows meningitis.

A most important factor influencing the course and type of the disease is the serum treatment. Absence of or inefficient serum treatment is responsible for fatal results either early in the disease or after a chronic illness due to adhesions or chronic septicaemia. The different types of cerebro-spinal fever show transitional forms, and description of the forms is apt to be artificial and confusing, as a single case may appear to belong to two. Further, the clinical picture of the disease is often abruptly changed hy unexpected alterations, such as improvemont or the onset of grave symptoms. The following forms of cerebrospinal fever may be briefly mentioned without a detailed description, and to avoid repetition the symptoms will be mainly dealt with subsequently: (1) Fulminating; (2) ordinary acute; (3) abortive; (4) chronic: (a) septicaemic; $(b)$ encysted or loculated meningitis.

The posterior basic menıngitis of infants is chronic encysted meningococcic meningitis in young infants, and its special features appear to depend on the early age of the patients rather than on any peculiarity in the infecting organism. More than twenty years ago Still showed that the disease was due to a micro organism almost identical with the meningococcus, and of late years it has become recognized that there is no constant bacteriological distinction between the meningocoocus of posterior basic meningitis and that of cerebro-spinal fever in later life. But Andrewes states that serologically the meningococci may be of a different strain from those of the epidemic disease in adults, and M. H. Gordon states that the neningococci in posterior basic meningitis do not differ serologicaliy from those in adult cases, except that some, which are more difficult to identify, are usually atypical specimens of his Group IV.: Among the cases called posterior basic meningitis half occur under six months of age, three-quarter's within the first year, and practically all under the age of two years, but there is no essential difference between the condition of closed meningococcic infection of the cerebral ventricles in infants and in adults. It may be well to mention the main elinical features differentiating the clinical form called posterior basic 
meningitis from ordinary cerebro-spinal fever in adults: 1. The chronicity of the disease, for acute cases of meningoeocic meningitis do not come under this heading. 2. 'The rarity of eruptions; among Hildesheim's 100 cases there was no example of a purpuric rash and herpes was present in two cases only. An ill-defined erythema occurs in a few cases. 3. Amaurosis or loss of vision without objective changes in the retina or optic nerve is common; it was noted in 37 out of Hildesheim's 100 cases. 4. 'I'he rarity of deafness and the occasional presence of auditory hyperacuity. 5 . The prominence of opisthotonos, due to the greater flexibility of the spinal column. 6. Joint infection appears to be usually periarthritic rather than intra arthritic, and may be associated with pseudo-glioma or the opacity of the vitreous caused by meningococcic invasion and imitating a glioma.

Mixed and Secondary Infections.

In addition to the meningococcus the cerebro-spinal fluid may show various mioro-organisms, such as the tubercle bacillus, the pneumococcus, the influenza bacillus, streptococci, and staphylococci. Among 339 cases in the navy there were 8 of this kind, 4 with streptococci, 3 with pneumococci, and 1 with tubercle bacilli; such cases are, uf course, usually fatal, and recovery occurred in one case only, with pneumococcic infection present at the first lumbar puncture.

The. commonest secondary infection appears to be pneumococcic; and it would appear that secondary pneumococcic infection depends mainly on the virulence of the pneumococcus, for in a previous series of 300 cases of meningococcic meningitis Netter and Salanier found four cases only of secondary pneumococcic infection.

Fitzgerald, who reports 12 cases of meningococcic meningitis, three of which were also infected with pneumococci, suggests that this secondary infection is commoner than is usually recognized, and quotes Mervyn Gordon's opinion that it occurs in 5 per cent. of the cases. He points out that unless the lumbar puncture fluid is bacteriologically tested on each occasion cases may easily be missed, and thus the real reason for ascribed failures of serum treatment be undetected. All the reported cases of combined meningococcic and pneumococcic infection, with the exception of three recorded by Netter and Salanier, proved fatal. Combined tuberculous and meningococcic infection of the meninges is very rare. Of the recorded cases Bériel and Durand accept eight only.

Streptococcic infection may spread from the lumbar puncture wound, be due to otitic infection, or arise without any obvious focus of infection.

\section{RAsHes.}

The incidence of rashes varies in different epidemics thus, in one of the earliest accounts of the disease North says that haemorrhages, which in 1806-7 marked almost every case, were rarely observed in 1808-9. Writing in 1911, Netter and Debré stated that eruptions have been, generally speaking, commoner in Britain and America than in Germany, and especially in France. In the years before the war rashes were very rare in sporadic cases in adults and were hardly ever seen in children, but during the increased prevalence since 1914 rashes have been common; thus, among 502 cases in the Royal Navy during the first four years of war there were 296, or 59 per cent., with rashes. In France also purpura has become more frequent during the war, and Netter has suggested that this may be correlated with Dopter's observation that whereas before the war the infecting strain of meningococcus was in 96 per cent. of cases Type A (Gordou's I and III), during the war 'Iype B (Gordon's II and IV) has become equally if not more important, and that the increased frequency of meningococcaemia is also thas explained.

Among 277 cases Netter had 19 of meningococcic purpura without meningitis, and states that it has become commoner, and that as bullae can be raised by friction over the purpuric areas more easily than in other forms, this may be utilized to determine the strain of the infecting organisms and so enable the corresponding serum to be selected.

Varieties of Rashes.

The cutaneous rashes accompany or closely follow the onset of the disease: They are seen on the first or second day of the disease and are comparable to the rose spots of enteric fever. There is almost always an interval of two to three days between the appearance of the initial rasl and of labial herpes in cases which show both.

The characteristic rash is haemorrhagic, and may be either small and petechial, resembling that in malignau endocarditis, or purpuric like that in the malignant form: of the exanthemata and acute lymphocytic leukaemia. The rash may be petechial or purpuric from the start, or the petechial character may supervene on an erythematous, papular, rose-spot, macular, or blotchy eruption. The erythematous rash has been described as transien and is seldom recorded. Occasionally the initial rash is described as urticarial.

The rose spots, papules, and petechiae are presumably embolic and evidence of the stage of septicaemia or bloor invasion. They are more prone to occur in parts exposed to friction, especially the joints; in some instances the haemorrhagic eruption may be partly vesicular, in others bullae may form over purpuric areas, and in one such case in the navy a pure culture of meningococci was obtained from the fluid. In rare instances ulceration may supervene in large haemorrhagic areas, and yet recovery follow. Trophic bullae may form on various parts of the body, such as the fingers, and, according to Fairley and Stewart, are sterile. These observers divide the rashes into (1) primary - namely, the purpuric, petechial, and macular, due to septicaemia; and (2) the secondary, which do not appear until after the third day of the disease and are regarded as toxic.

\section{Herpes.}

Herpes is a well-recognized event in cerebro-spinal fever and may be considered under the two heads of (1) ordinary febrile herpes labialis, and (2) herpes of the zoster type.

Herpes labialis appears to occur with varying frequency in different epidemics, and the relative incidence of herpes labialis and of other cutaneous eruptions shows similar fluctuations; from a review of the literature up to 1898 , Councilman, Mallory, and Wright concluded that herpes is far commoner than any other eruption. Netter found that herpes occurred in a third of his cases, and was more frequent than purpura, and Sainton states that it was present in two-thirds of his cases. On the other hand, Stille says that in the Massachusetts epidemic herpes was infrequent, and much less often present than cutaneous rashes; in 502 naval cases there were.117, or 23 per cent., with herpes, and 296 , or 59 per cent., with rashes.

Labial herpes appears later than the initial rash. and usually on the fourth day of the disease, but it may occu. earlier, on the second day, or be postponed until later. In rare instances it may recur during continued fever, with a recrudescence of symptoms, or with the appearance of some complication. It is unusual in children, and exceptional in infants.

The causation of ordinary herpes labialis is undecided; it is not generally thought to be associated with any lesion of the central nervous system, though Howard, from histological examination of the Gasserian and other ganglia considers that the herpes of pneumonia and cerebro-spinal fever has a similar anatomical basis to that of herpes zoster. Though frequently looked for, meningococci have very seldom been isolated from the vesicles of labial herpes. It has been suggested that the vesicles are due to meningococci, and their situation determined by inflam. mation of the Gasserian ganglion. As herpes labialis has been seen in persons reacting vigorously to prophylactic antimeningococcic vaccines it appears reasonable to refer it to the local action of toxins rather than of bacteria in the skin.

Herpes of the zoster type occurs in cerebro-spinal fever, but much less often than Iabial herpes. The two forms may be present at the same time; thus labial herpes and herpes of the external ear due to inflammation of tho geniculate ganglion of the facial nerve, or labial herpes and herpes of the neck may be combined.

Nervous Symptoms.

Nerrous symptoms are predominant at the onset of meningitis; severe headache, vomiting, and mental stupo or delirium are usually present, and there may be wild mania, symptoms suggesting delirium tremens, or epileptic 
seizures. The mental disturbancos during the septicaemic stzues, and before meningeal infection his occurred, are probably toxic, and comparable to those at the commenceiuent of other acute infections, such as pneumonia. Jucontinence of urine and faeces is common. Swallowing may be difficult, either from oesophiageal spasm or paresis, and is probably more frequent than the notes of cases show. Among the 502 naval cases it was mentioned in 8 (5 fatal). In a case recorded by McConnell, Morris, and Seehorn oesopbageal spasm was so obstinate that gastrostomy was done, death following twelve hours later. General hyperaesthesia is not uncommon.

The condition of the reflexes varies; the deep reflexes are often exaggerated and an extensor response may be present; among 398 naval cases Babinski's sign was noted in 35 , or 8.8 per cent.; it may be obtained on one side only. Herrick insists that whereas in other acute infections the leep reflexes may be equally exaggerated on the two sides, in cerebro-spinal fever there is a notable inequality. Kiernig's sign and rigidity of the neck are practically constant when meningitis has appeared, and are so well known that they will not be further discussed here.

Facial paralysis is rare; it appears early and is transient. Among 502 naval cases it was recorded in 7 , but among Robb's 230 cases there were 11 examples.

\section{Paraplegia.}

Paraplegia is rare; among 502 naval cases it was noted in 1 ; among more than 400 cases MacLagan found it in 3,2 of which recovered; there was one example among Robb's 230 cases, and none among 120 cases that recovered and were analysed by Worster:Drought. It may be either organic or, in very rarely recognized instances, functional.

Organic paraplegia is described by Sophian as being either (1) spastic, with ataxia, clonus, extensor plantar response, no sensory changes, and no vesical disorder ; (2) flaccid, with absence of reflexes and sensory changes. of the spastic form Sophian has seen 12 cases, presumably due to the pressure of exudate around the cord some way above the cauda equina. The general experience is that the paraplegia slowly improves; but in some instances it is permanent.

Functional Paraplegia. - In convalescents the gait is naturally at first impaired, and as the patients may be unduly susceptible to auto-suggestion hysterical paraplegia may result.

Hemiplegia: Monoplegias: Nerve Deafness.

Hemiplegia is rare, and, like paraplegia, may be organic or functional. Among the 502 naval cases it occurred in 12 , 10 of which proved fatal. Robb found 4 cases of hemi. plegia among 230 cases at Belfast.

Mionoplegias of the limbs due to inflammation of the nerve roots (radiculitis) as they pass through the meninges are described by Netter and Debré. 'They are even rarer' than hemiplegia, occur earlier, are accompanied by wasting, pain, loss of reflexes and of sensation, but they usually disappear. Some only of the muscles of the linb may be affected. Sophian, however, states that monoplegias occur late in the course of the disease.

Nerve decifness is not nearly so common now as it appear's to have been formerly. Deafuess may also be due to otitis media, which is not a commonly recognized com. plication of cerebro-spinal fever; among 502 naval cases it was noted in 10. But some writers state that a mild form of otitis media is very common.

Ocular Signs and Symptoms.

The pupils are usually dilated, due to irritation of the sympathetic, and when sluggish in addition point to increased intracrauial pressure.

Photophobia, said to be rare, and thus to contrast with its frequency in tuberculous meningitis, was reported in 52 , or 10 per cent., out of 502 naval cases, and was almost always an initial symptom.

Conjunctivitis is not very common; it occurred in 5.6 per cent. of 502 naval cases, in 10 (or 9 per cent.) of Councilman, Mallory, and Wright's 111 cases, in 15 (or 20 per cent.) of Ballantyne's 73 cases, and in 20 per cent. of Fairley and Stewart's series. The pus may, but does not al ways, contain weningococci. Conjunctival haemorrhages are sometimes present, and are important diagnostically, as they are practically never seen in other forms of meningitis.

Panophthalmitis is rare and is commonly unilateral; Lery (of Essen) had the exceptional experience of seeing 9 cases, 8 unilateral, among 165 cases of the disease. Among the 502 naval cases it occurred in 7 , or 1.4 per cent., and was bilateral in 2 and unilateral in 5 , all on the right side; in this connexion attention may be drawn to Netter's suggestion that the position of the patient's head on the right or left, determines the side on which unilateral panophthalmitis occurs. As long ago as 1867 S. Gondon noticed the predominance of these infective conditions in the right eye.

\section{Optic Neuritis and other Nerve Lesions.}

Optic neuritis is usually regarded as infrequent and thas contrusting with its incidence in tuberculous mening. itis; it seems to be specially related to septicaemic infection.

True nystagmus occurs in severe cases only, and by Fairley and Stewart is regarded as pathognomonic of internal hydrocephalus, and therefore of extremely grave prognosis. It must : be distinguished from pseudo nystagmus or jerky movements at the limit of fixation, which, according to Ballantyne; are without significanoe.

Strabismus, usually due to implication of the: sixth nerve, is much less frequent than in tuberculous mening. itis, and is usually transiont.

Ptosis is much less frequent than strabismus; ont of the 502 naval cases it was noted in 18 , or 3.6 per cent but 13 or 72 per cent., of these 18 cases terminated fatally. The mortality was thus 20 per cent. higher than in the squint cases; this may be correlated with the probable spasmodic nature of many of the squints and the paralytic origin of the ptoses.

Retraction of the upper eyelids may occur; among 73 cases Ballantyne noted it in 15, one of which also showed von Graefe's sign.

Circulatory Symptoms and Complications.

Pericarditis is probably often latent, as it is found after death when previously unsuspected - an experience familiav in other conditions. Nutter and Debré speak of pericarditis as a phenomenon almost sonfined to the post-mortem table and some authors do not refer to it. Robb, however, noted pericardial friction in 17, or 7.4 per cent., out of 230 cases.

Endocarditis is probably not so rare as it has been stated to be, but the vegetations may be small and possibly are sometimes overlooked.

Phlebitis in the lower extremities has been reported in rare instances.

The blood shows a polymorphonuclear leucocytosis of from 20,000 to 50,000 , and, according to Koplik, is over 25,000 in more than half the cases; in chronic cases the leucocytosis may fall to normal.

Pulse and Blood Pressure.

The pulse is usually regular, though it may, especially in grave cases, be irregular. Like the temperature, the pulse rate may vary greatly within a short time. The outstanding feature about the pulse is that it is so often slow in relation to the temperature; thus, in a naval chaplain who was maniacal in the early stage the tempera. ture was $105^{\circ} \mathrm{F}$. and the pulse 60 . The slow pulse depends on ragal inhibition due to increased intracranial pressure. In fulminating and septicaemic cases the pulse is rapid, as in most other acute infections. Before death the pulse may become extremely rapid.

The arterial blood pressure is low in the septicaemic stage, and in the worst cases may be impossible to record, rises with the increased intracranial pressure accom. panying the onset of meningitis, and may then be between 140 and $190 \mathrm{~mm}$. $\mathrm{Hg}$, and in internal hydrocephalus is almost always raised from pressure of the cerebro-spinal fluid on the floor of the fourth ventricle. During convalescence the blood pressure is, in the absence of complications, normal or subnormal. The tache cerebrale is usually well marked, but is not of any diagnostic value. Epistaxis is occasionally seen.

\section{Temperature.}

The temperature is very irregular and does not conform to any rule; probably every case has fever at some time 
during the course of the disease, but some charts show little or no elevation of the temperature. There may be extreme oscillations at very short intervals-a feature of some diagnostic value in the early stages, according to Netter.

Complicationg in Thorax and Alimentary Stytem.

Pulmonary complications in this country since the war have not been very common; bronchitis and broncho. pneumonia have been the most frequent and important.

Pleurisy may; of course, accompany pnenmonia or bronchopneumonia in the course of cerebro-spinal fever, but it may occur without any obvious lung lesion; this occurred in 3 out of 502 naval cases.

Parotitis is rare; it may be due to an ascending infection from the mouth; as in other fevers and conditions in which the mouth is dry:

In the most severe cases there may be blood in the vomit and faeces, but rarely in considerable quantities. Diarrhoea is not uncommon as an initial symptom, and, as mentioned olsewhere, abdominal symptoms may be so prominent as to saggest an acute perforation of the alimentary tract, appendicitis, or Henoch's purpura. . Jaundice is quite exceptional, and one case of peritonitis is tabulated by Herrick.

\section{Lesions of Joints.}

Arthralgia, or pain in the joints like that of influenza, is common at the onset and in the early stage of the disease, but is often overshadowed by the more severe symptoms.

Arthritis, or more often synovitis, is a recognized com. plication of cerebro-spinal fever, and may occur in meningococcic infection without meningitis, and in such cases has sometimes been regarded as "peliosis rheumatica."

The incidence was estimated at from 10 to 15 per cent. by Sophian, and from 5 to 20 per cent. by Roger, but among 502 cases in the navy it occurred in 24 , or 4.8 per cent., and among Fairley and Stewart's 323 cases in 23, or 7 per cent. Among 902 other cases there were 59 cases of synovitis, or 6.5 per cent. It is more often seen in adolescents and adults than in babies, thus recalling the incidence of synovitis in acute rheumatism; but when it does occur in babies the hands and feet are specially picked out, whereas in older patients the larger joints-the knees, wrists, and ankles-are usually attacked. In posterior basic meningitis Still found periarticular infection, the joint cavities being healthy in 4 out of 49 cases examined after death, and Osler, in his Cavendish lecture, said the lesions were generally periarticular.

Clinical Features of Joint Lesions : Prognosis.

The accounts of the clinical features of the joint lesions in cerebro-spinal fover vary; thus, by some writers the joints are said to be very painful, by others as tolerant of imovement.

The prognosis as regards the condition of the joint is good; the early cases clear up rapidly and commonly with. out any necessity for the intra-articular injection of serum. Even suppurative cases may clear up after simple aspira. tion. Ankylosis, of which Roger records a case with implication of the hip and knee, is rare.

The chronic cases of meningococcic pyarthrosis may initate gonococcic or tuberculous arthritis. When the synovitis occurs about the eighth day of the disease it may be difficult to decide whether it is meningococcic or the first sign of serum disease. For the treatment intra-articular injection of serum has been widely recommended, but most cases clear up with purely symptomatic treatment. Salicylates do not exert any influence on the synovitis, but aspirin relieves the pain.

\section{Urinary Changes.}

The urinary changes are not characteristic or of clinical importance.

Cystitis may occur, and the incidence of pyuria has varied in different outbreaks. Pyelitis was reported in 5 per cent. of the Texas epidemic by Sophian, who described meningococci in the urine, but general experience shows that meningococci are rare in the urine, and that cystitis and pyuria during the disease may be due to other organisms; polyuria is occasionally noted.

Albuminuria is not very common, and when present is usually small in amount.
Haematioria is not common; when it occurs it usually is an early complication, and is associated with a petechial or haemorrhagic rash; it is presumably associated with haemorrhages in the mucous membrane of the urinary tract, but is not so often seen in the fulminating cases witi much purpura as might naturally be expected. Among the 502 naval cases it was noted in 4 only.

Glycosuria is rare and may be quite transient. It is stated to be less frequent than in tuberculous meningitis, in which it occurs late; Garrod and Frew found it in 15 out of 41 cases of tuberculons meningitis, but never in posterior basic meningitis. The glycosuria would appen. to be nervous and central in origin, but more than this it is difficult to say.

Urobilinogenuria was recorded by Cazamian in threequarters of his 113 cases; it was most intense at the onse and was as frequent in the non-septicaemic as in the septicaemic cases. Indicanuria is stated to occur in grave cases.

\section{EPIDIDYMitis AND ORCHITIS.}

Epididymitis and orchitis are complications of cerebro spinal fever, and the meningococcus has been obtained by puncture of the inflamed organ, but it is rarely mentioned in the textbooks.

It appears that both meningococci (Gordon's Types I and III) and para-meningococci (Gordon's Types II and IV) may be associated with orchitis and epididymitis; but it is interesting that Latham's cases were associated with Type IV, which seems particularly prone to cause septicaemic and metastatic lesions.

It is generally considered that the epididymitis and orchitis are septicaemic in origin. I have not any reference to cases in boys under the age of puberty. In about 10 per cent. of the cases the lesion is bilateral. Orchitis or epididymitis is almost always transient, subsiding without suppuration and not being followed by atrophy.

Relapses and Recrudescences.

There is some confusion between these two terms, and therefore some uncertainty about the frequency of true relapses.

Recrudescences, sometimes called intermittent relapses, or the return of symptoms before the patient has really recovered from the disease, are very common, and a patient may have several; among the naval cases one patient had 7, and Ker quotes 13 recrudescences.

True relapses are rare. Netter appears to draw the line between recrudescences and relapses at a month from the disappearance of symptoms, and reported 4 , or 1.6 per cent., among 255 cases.

Late relapses at long intervals after the patient has been cured can hardly be distinguished from second attacks. These cases of second attack are very rare; it is noteworthy that one attack of meningococcic infection usually protects against another, and that, as Adami has pointed out, the meningococcus thus differs from the majority of pathogenic micrococci.

\section{Diagnosis.}

For the certain diagnosis of meningococcic meningitis bacteriological examination of the cerebro-spinal fluid for meningococci is necessary, and similarly in the premeningitic stage of cerebro-spinal fever a blood culture is essential. No doubt genuine cases of cerebro-spinal fever may be ruled out by failure of bacteriological methods to give positive results. But this error is probably much less than that which would result from the inclusion of cases diagnosed on clinical grounds, and in the latter event it would be difficult to know where to draw the line. Cases with meningitic symptoms and meningococci in the nasopharynx but not in the cerebro. spinal fluid, though often probably genuine cases, and, indeed, so regarded by Flack, are open to the criticism that they may be meningococcus carriers with meningitis or meningism due to some other cause.

- No clinical manifestation is pathognomonic of meningo. coccic infection. A haemorrhagic rash, though highly suggestive of meningococcaemia, may be present in pneumococcic, streptococcic, and influenzal infections, and in malignant forms of the exanthemata, such as gmall. pox. From other forms of meningitis, such as tuberculous, 
pneumococcic, otitic, influenzal, an undoubted diagnosis can be made only by lumbar puncture and examination of the cerebro-spinal fluid.

As lumbar puncture is such an essential element in the diagnosis, it is well to insist that the risk of any harm from diagnostic puncture, provided the fluid is not withdrawn too rapidly or in excessive quantities, is almost negligible; some haemorrhage may occur, but this seldom causes serious damage.

The risk of introducing infection and setting up mening. itis by diagnostic puncture may be practically dismissed, and is quite different from the danger of infection from repeated tappings.

Although it is simple enough to depend for the diagnosis of meningococcic meningitis on the examination of the cerebro-spinal fluid for meningococci, there are a number of cases in which a decision is still left in doubt. Cases certainly occur about which the purely clinical diagnosis appears unquestionable, but in which the cerebro-spinal Huid, though turbid from the presence of polymorphonuclear leucocytes, does not contain any micro-organisms. 'The occurrence of cases with a sterile polymorphonuclear fluid at the first lumbar puncture recalls Hort's contention that the meningococcus is only one of the phases in the life-cycle of the virus, and raises the unorthodox suggestion that in some of the phases the organism may be a filter-passer.

These cases should be treated, although they cannot be tabulated, as meningococcic; recovery is in favour of a meningococcic origin, not only from the point of riew of this therapeutic test, but also because other forms of poly. morphonuclear meningitis are usually fatal, recoveries in pneumococcic meningitis being most exceptional.

As already mentioned, the cerebro-spinal fluid, though characteristically polymorphonuclear, may, particularly in chronic cases, show a predominance of lymphocytes, and so resemble the cytology of the meningitis of tuberculosis, syphilis, mumps, malaria, acute lead poisoning, and of the meningitjc form of acute poliomyelitis. Incidentally, some of the clinical differences from tuberculous meningitis have been referred to, and with regard to the others, though it is an interesting academic study, lumbar puncture will always be necessary, and more rapidly and surely decide the diagnosis and prognosis.

Meningism may be due to such a large number of acute infections that it would take too long to detail them. In particular, the lecturer mentioned pneumonia, otitis media, influenza, malaria, and salvarsan injection in this connexion.

Discussing the differential diagnosis, the lecturer mentioned tonsillitis, measles, rubella, rheumatic stiffness of the neck, and rheumatic fever as possible causes of difficulty.

Spirochaetosis ictero-haemorrhagica may cause mening.

itis, and in some of these cases there may be little or no jaundice.

In the acute infective polyneuritis described by Bradford, Bashford, and Wilson, the initial symptoms of headache, vomiting, pain in the back, and moderate fever, are in rare instances sufficiently severe to suggest the possibility of cerebro-spinal fever, but lumbar puncture shows that there is not any meningitis. Anthrax with cerebral symptoms may imitate meningococcic meningitis.

The meningitic form of Acute Poliomyelitis.-Clinically the resemblance to cerebro-spinal fever is very close; thus in 1911 Reece found that a reputed outbreak of cerebrospinal fever was really one of acute poliomyelitis without any admixture of meningococcic cases.

Encephalitis lethargica, when first seen in Paris and elsewhere, was thought to be meningococcic meningitis until lumbar puncture put this diagnosis out of court. The cerebro-spinal fluid is clear and the cell content usually normal; if there be any cytological change it is in the direction of a lyinphocytosis. Another difference from meningococcic infection is the absence of a haemic leucocytosis. The striking features of the clisease-lethargy and ocular paralyses, though the latter are not constantshould arouse suspicion.

Uraemia may be suggested by fulminating cases in an unconscious condition, especially as there may be albuminuria, or from collapse, suppression of urine, and petechial rash which might be thought to be uraemic. In pregnant women convulsions during cerebro-spinal fever have been regarded as eclamptic.

The purpuric eruption of the fulminating and acute cases may lead to confusion with fulminating and other forms of purpura, such as acute lymphocytic leukaemia, streptococcic septicaemia, Henoch's purpura, and the diagnosis may be cleared up only after death. Haemorrhagic small. pox occurring in connexion with cerebro-spinal fever may be regarded as the meningococcic infection. In the pas the disease appears to have been confused. with malignant measles, for in 1867 Gordon stated that haemorrhagic measles always accompanied cerebro-spinal fever.

Typhus, severe food poisoning, and acute osteomyelitis were also considered.

\section{HOW TO READ STATISTICS.} BY

A. S. PERCIVAL, M.A., M.B., B.C.CAMB., NEWCASTLE-ON-TYNE.

IT is a common but erroneous saying that statistics can prove anything; invaluable logical deductions can be drawn from complete statistical tables, but the conclu. sions drawn from incomplete tables are always fallacious. Every week one sees from insufficient data conclusions airily drawn that convince the simple reader; even the masters of the profession fail in this way: Gowers pointed out that among the lower classes 80 per cent. the tabetics have been infected with syphilis, and therefore the preponderating cause of tabes was syphilis. Many people are so overwhelmed with a bald statistical state ment of this sort that they seem to lose all their reasoning power. A moment's thought will show that the only logical conclusion that can be drawn from such a bald statement is that some syphilitics suffer from tabes. It quite probable that less than 4 per cent. of syphilitics tabes. It is not my intention to dispute the relation of tabes to syphilis, but to show what data are required before any association between two attributes can bo inferred from statistical evidence.

Suppose that the following statement is made, and that it is known to be true of a certain assemblage: "99 cent. of those who had red hair were Canadians." How many are apt to infer from this that the majority of Canadians had red hair! But a little thought will show that from the premiss it is impossible to say whether there is any asso. ciation between Canadians and red hair; it is quite possible that 99 per cent. of the Canadians there had not red hair, as will be easily seen from the ad. joining diagram.

Let the number of Canadians present be represented by the larger circle

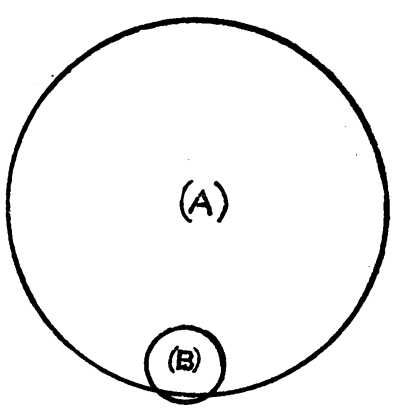
(A) and the number of those

with red hair by the smaller circle (B). Let $(\mathrm{N})$ denote the total number of observations, or in this case the total number of inhabitants in the place considered, say $(N)=12,000$. Let capital letters denote the attributes to be investigated -for example, A denotes Canadians, B red hair. Further, let the number of those who possess the attribute of $\mathrm{A}$, or the "class-frequency" of $A$, be denoted by (A); that is, let the class-frequency of an attribute be denoted by the attribute symbol enclosed in brackets. Similarly, let the class-frequency of the "not-A's" be denoted by $(a)$, the corresponding small letter enclosed in brackets. Now suppose that the following five class-frequencies are given :

$$
\begin{aligned}
& (\mathrm{N})=12,000 \\
& (\mathrm{~A})=11,880 \\
& (a)=120
\end{aligned} \text { and } \quad \begin{aligned}
(\mathrm{B}) & =100 \\
(\mathrm{~b}) & =11,900
\end{aligned}
$$

Of course it is always true that $(\mathrm{N})=(\mathrm{A})+(a)=(\mathrm{B})+(b)$, so that two of these data are redundant. For instance, if (A), (a) and (B) are given, (N) can be obtained by adding (A) $+(a)$; and $(b)$ by finding the difference $(\mathrm{N})-(\mathrm{B})$. The above data are not sufficient to enable one to draw any conclusion as to the association of Canadians and red hair. For that we must know either how many Canadians have red hair-that is, the class-frequency (A B), or the class-frequency $(\mathrm{A} b)$, or some other associated classfrequency such as $(a b)$. 